\title{
Dynamic changes in the zone of apposition and diaphragm length during maximal respiratory efforts
}

\author{
D K McKenzie, S C Gandevia, R B Gorman, F C G Southon
}

\begin{abstract}
Background - Clinical tests of diaphragmatic strength are limited by the wide normal variation in maximal pressure which result, in part, from changes in diaphragmatic length. During relaxation at different lung volumes diaphragmatic length (LDI) can be estimated from the length of the zone of apposition (Lzapp) and the transverse diameter of the rib cage (DRC). A study was carried out in two subjects using sequential digital radiography at six frames/second to determine whether these relations apply during maximal respiratory efforts which distort the rib cage and diaphragm.
\end{abstract}

Methods - The length of the anteroposterior contour of the diaphragm and DRC were determined by curve fitting. Lzapp was measured with a millimetre rule.

Results - A significant correlation was found between LDI and Lzapp during both maximal inspiratory and expulsive manoeuvres $\left(R^{2}=0.88\right.$ and 0.52$)$. LDI was estimated from the measurements of Lzapp and DRC using a multiple regression equation derived from measurements during static relaxation. Despite the complex dynamic events at the onset of these "static" manoeuvres, actual LDI correlated strongly with derived LDI using all data for the two manoeuvres in each subject $\left(R^{2}=0.95\right.$ and 0.84$)$. Measurements with ultrasonography (12 cm linear probe) and magnetometers confirmed the changes in Lzapp and DRC during inspiratory and expulsive efforts. Conclusions - Non-invasive measurements of Lzapp and DRC can be used to derive an accurate estimate of diaphragmatic length under dynamic conditions.

(Thorax 1994;49:634-638)

The range of normal values for maximal transdiaphragmatic pressure extends almost 10 fold (from 30 to $300 \mathrm{~cm} \mathrm{H}_{2} \mathrm{O}$ ) because of variability between subjects and between different test manoeuvres. ${ }^{1}$ To date there have been limited attempts to account for the sources of this variability in order to develop a more precise clinical test of respiratory muscle strength. ${ }^{2}$
Maximal pressures developed by the diaphragm depend, not only on its neural drive and muscle strength, but also on its forcelength and force-velocity relations. Other factors, including the cross sectional area of the lower rib cage and diaphragmatic shape, may also be important. Using sequential digital radiography at six frames/second we observed progressive muscle shortening at the onset of maximal "static" inspiratory efforts which move the diaphragm to a less advantageous position on the force-length curve. ${ }^{3} \mathrm{By}$ contrast, the "extra" pressure developed during expulsive manoeuvres with the glottis held open was associated with transient lengthening of the diaphragm ${ }^{4-6}$ produced by the abdominal muscles. ${ }^{78}$ The changes in length and velocity of diaphragmatic muscle explained much of the difference in maximal voluntary transdiaphragmatic pressure between the manoeuvres. ${ }^{3}$ These observations suggest that measurements of the diaphragmatic muscle length and velocity might improve the predictive power of clinical tests of respiratory muscle strength.

Because diaphragmatic length (LDI) is difficult to measure in vivo, interest has focused on the relation between LDI and the width of the zone of apposition between the diaphragm and chest wall (Lzapp). A linear relation exists between LDI and Lzapp (measured from radiographs) during relaxation at lung volumes between residual volume (RV) and total lung capacity (TLC). ${ }^{9}$ During an "isovolume manoeuvre", however, in which LDI is essentially constant, Lzapp decreases as the diameter of the rib cage (DRC) increases because the diaphragm "peels away" from the chest wall. ${ }^{1011}$ Rochester and coworkers ${ }^{12}$ examined radiographs taken during relaxation at different lung volumes and found that $95 \%$ of the variance of Lzapp can be accounted for by measurements of LDI and the transverse diameter of the rib cage (DRC). Further studies in anaesthetised dogs showed that Lzapp correlated well with LDI during unloaded respiration. ${ }^{13}$ The authors concluded that measurement of Lzapp may be invaluable in the study of breathing mechanics. However, these measurements have not been reported for the strong respiratory efforts used to test diaphragmatic strength and endurance in human subjects. ${ }^{2}$ There are substantial distortions of

Received 5 August 1993 Returned to authors 28 January 1994 23 February 1994 Accepted for publication 1 March 1994 
the rib cage $\mathrm{e}^{1415}$ and diaphragm ${ }^{6}$ during these efforts, and these might influence the relations between Lzapp, LDI, and DRC.

The present study was designed to examine the correlated changes in Lzapp and DRC during maximal voluntary efforts. Measurements were made with sequential radiography and confirmed with ultrasonography.

\section{Methods}

Because the experiment involved exposure to radiation of more than 10 millisieverts ( $\mathrm{mSv}$ ), only two subjects (authors) were used (DM, subject 1,39 years, $189 \mathrm{~cm}, 76 \mathrm{~kg}$; SG, subject 2,36 years, $172 \mathrm{~cm}, 62 \mathrm{~kg}$ ). Both were trained in the respiratory manoeuvres so that the lowest exposures could be achieved (see below). The recording procedures were approved by the institutional ethics committee. All radiological procedures were supervised by the radiation protection physicist from our institution (GS), and informed consent was obtained. The methodology has been reported in detail elsewhere ${ }^{3}$ and is described briefly here.

\section{RECORDING PROCEDURES}

Oesophageal, gastric, and transdiaphragmatic pressures were separately recorded with a single catheter. ${ }^{7}$ Mouth pressure was also measured proximal to a shutter. An $x$ ray detector on the abdominal wall provided a timing signal to allow precise temporal correlation between the radiographic images and physiological data. All analogue signals and the timing signal were recorded on tape (DC, $1.25 \mathrm{kHz}$ )

The maximal inspiratory efforts were performed against a shutter with minimal elevation of abdominal pressure. During expulsive efforts the subject attempted to keep the glottis open. Each manoeuvre began at a relaxed end expiratory position and was repeated six times.

Radiographs were obtained using a digital angiography unit (Phillips DVI) equipped with a $35 \mathrm{~cm}$ image intensifier. The subject was seated and markers were taped over the spine and the ninth interspace in the mid axillary line. Images were obtained at six frames/second with anteroposterior and lateral beams (11-16 frames in each contraction). Only the anteroposterior images were used for this study. In subject 2 anteroposterior images enclosed the full width of the lower chest and upper abdomen. In subject 1 the field enclosed only the left half of the chest and abdomen so measurements were doubled to give approximate LDI and DRC. ${ }^{12}$ Images were printed with minimal distortion on $10 \times 13.5 \mathrm{~cm}$ film.

Values for the pressures were measured at the onset of each radiation pulse (duration $20 \mathrm{~ms}$ ). To digitise the diaphragm profile a grid was placed on each radiograph overlying a spinal marker and six points along each hemidiaphragm were resolved into cartesian coordinates. ${ }^{3}$ Coordinates for the lateral costal origins of the diaphragm were measured from anatomical landmarks defined at TLC. Lzapp was assumed to be zero at TLC. ${ }^{12} \mathrm{~A}$ curve was fitted by computer so that the total length of the diaphragm (or hemidiaphragm) could be calculated. Coordinates were adjusted for display and imaging scaling. We assumed that one quarter of the resting length comprised an inextensible tendon. ${ }^{39}$ The length of Lzapp was measured from the same images using a transparent rule graduated in millimetres. The diameter of the rib cage (DRC) was derived for each frame as the widest horizontal distance encompassed by the curve fitted to the diaphragm coordinates.

Ultrasonography was used to assess changes in Lzapp in the same subjects in separate studies conducted after the radiographic imaging (Accuson XP-128). DRC was measured with linearised magnetometers attached to the skin in the mid axillary line by double-sided tape. Measurements were made using a $12 \mathrm{~cm}$ linear array probe $(3.5 \mathrm{MHz})$ held firmly against the rib cage in the longitudinal axis just anterior to the magnetometer in the right mid axillary line. All images were stored on video tape together with a simultaneous signal of PDI. Oesophageal and gastric pressures and PDI and DRC were also recorded on magnetic tape. The length of the probe ensured that the full extent of Lzapp could be followed throughout each manoeuvre (at 18-28 frames/ second). Before each maximal manoeuvre the subject inhaled to TLC so that the costal insertion of the diaphragm could be visualised. Other methods were similar to those of the radiographic study. Images were replayed and
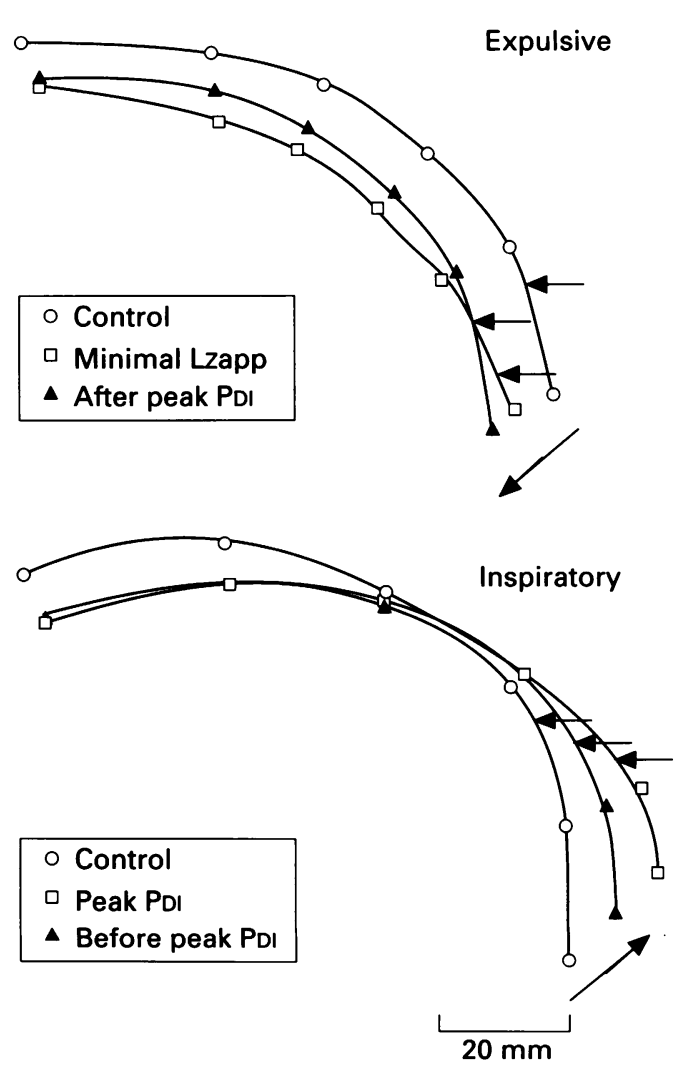

Figure 1 Representative profiles of left hemidiaphragm (posteroanterior projection) of subject 1 showing changes in shape and position during expulsive and inspiratory manoeuvres. Horizontal arrow heads indicate length of zone of apposition from insertion (lowest symbols). Diagonal arrows show direction of motion of diaphragm costal insertion during manoeuvres. 

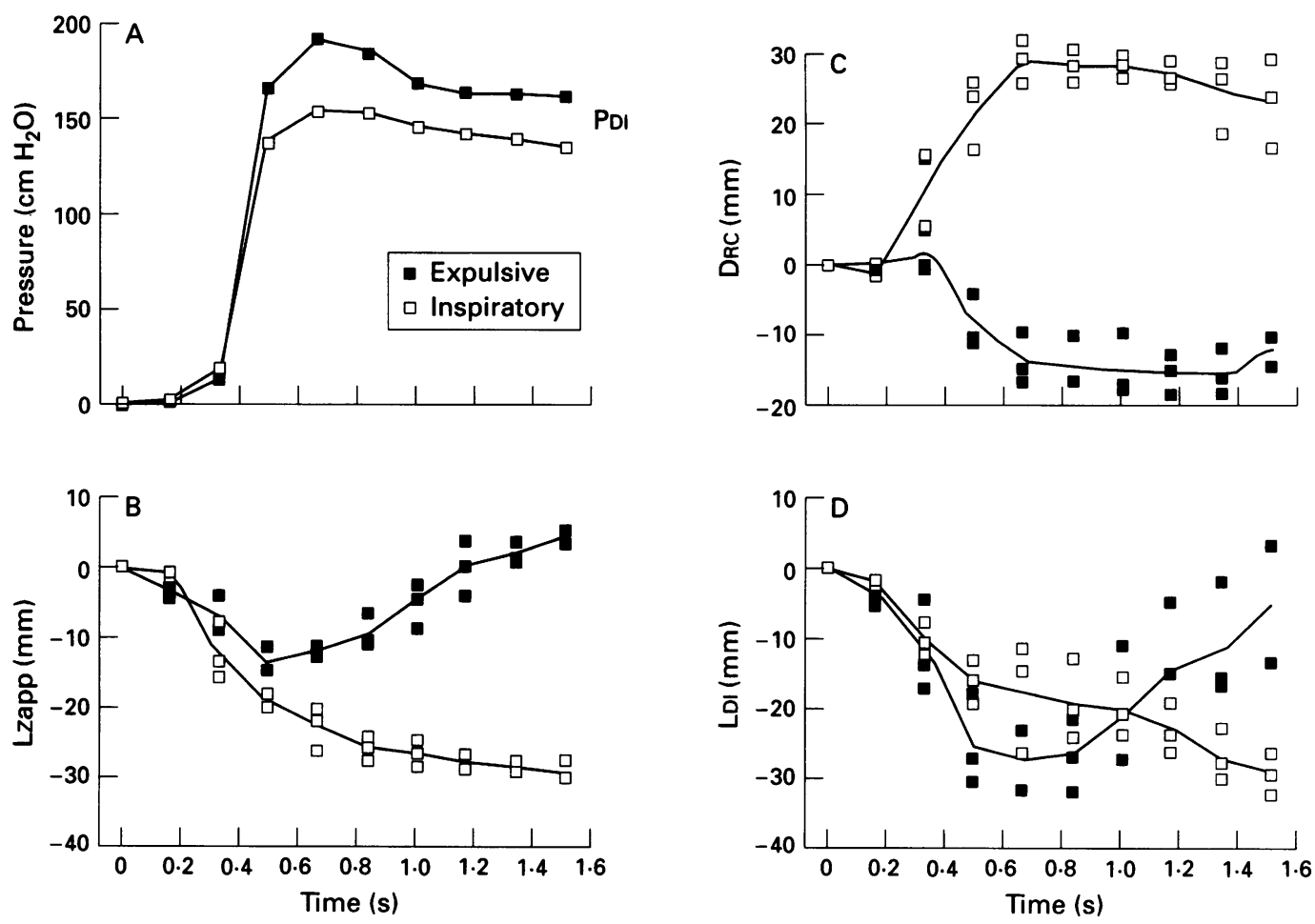

Figure 2 Data from subject 1 showing $(A)$ values for PDI during representative maximal expulsive and inspiratory efforts. Panels $B, C$, and $D$ show all data points for the relative changes in the length of the zone of apposition (LZapP), transverse diameter of the rib cage (DRC), and diaphragm length (LDI) from the three efforts in each manoeuvre (lines indicate mean values).

measured on a high resolution screen. Approximately one in four images was measured so that the overall sampling rate was comparable to that for the sequential radiography (six frames/second).

\section{Results}

The radiographic study showed that both diaphragmatic manoeuvres were associated with complex changes in Lzapp, LDI, DRC, and dome shape (fig 1). The manoeuvres differed in the direction of motion of the origin of the diaphragm, in the shape of the dome, and in the changes in Lzapp. The lateral costal margins moved outwards and cephalad at the onset of the inspiratory manoeuvre whereas, during the expulsive effort, they moved inwards and caudad. The dome became flattened with greater radii of curvature during the development of maximal inspiratory pressure, whereas the expulsive manoeuvre produced a more rounded shape with a reduction in radii of curvature.

All data for PDI, Lzapp, and DRC are shown for subject 1 in fig 2 . There was progressive shortening of the diaphragm at the onset of the inspiratory contraction with a considerable decrease in Lzapp. The absolute decrease in Lzapp (22 $\mathrm{mm}$ to the peak pressure) was more than twice the reduction in length of the hemidiaphragm. This was due to the outward motion of the rib cage (increase in DRC) which resulted in "peeling away" of the diaphragm from the chest wall independent of LDI (see also fig 1). During inspiratory efforts in subject 2 , however, the decrease in Lzapp was almost identical to the decrease in hemidiaphragm length $(25 \mathrm{~mm})$ because there was minimal change in DRC in two of the three trials.
Diaphragmatic motion during pressure development in the expulsive manoeuvre was biphasic. During the initial phase (to about $70 \%$ PDImax in the expulsive effort) there was progressive shortening of Lzapp and LDI in both subjects. The subsequent pressure development (above that in the inspiratory manoeuvre) was associated with an increase in Lzapp and LDI. In subject 1 the increase in Lzapp preceded that of LDI whereas, in subject 2, both parameters changed simultaneously. This may reflect differences in the timing of abdominal muscle recruitment or in rib cage compliance.

CORRELATIONS BETWEEN LZapp, LDI AND DRC The regression equations relating Lzapp to LDI and DRC in each subject are given in the table. There was a significant correlation between LDI and Lzapp in both subjects for both manoeuvres. In the dynamic phase of these manoeuvres, however, the relation between DRC and Lzapp was variable, both between subjects and the manoeuvres. In sub-

Regression equations relating Lzapp to LDI and DRC

\begin{tabular}{cll}
\hline & Regression & $R^{2}$ \\
\hline Expulsive & & \\
Subject 1 & Lzapp $=-128+0.464 \times$ LDI & $0.624^{* *}$ \\
& Lzapp $=30-0.059 \times$ DRC & 0.004 \\
Subject 2 & Lzapp $=-235+0.742 \times$ LDI & $0.419^{* *}$ \\
& Lzapp $=304-1.069 \times$ DRC & $0.532^{* *}$ \\
& & \\
Inspiratory & Lzapp $=-318+1.004 \times$ LDI & $0.887^{* *}$ \\
Subject 1 & Lzapp $=244-0.826 \times$ DRC & $0.804^{* *}$ \\
Subject 2 & Lzapp $=-123+0 \cdot 400 \times$ LDI & $0.780^{* *}$ \\
& Lzapp $=282-1.031 \times$ DRC & $0.156^{*}$ \\
\hline
\end{tabular}

Lzapp = length of the zone of apposition of the diaphragm; LDI = length of the diaphragm (anteroposterior projection); DRC = maximal diameter of the rib cage (anteroposterior projection). All values in mm. 
ject 1 there was an inverse correlation during the inspiratory manoeuvre but no significant relation during the expulsive manoeuvre. In subject 2 the inverse correlation was better for the expulsive than the inspiratory manoeuvre. This reflected the small changes in DRC in this subject, especially for the inspiratory manoeuvre.

To determine whether LDI during maximal respiratory manoeuvres could be reliably estimated from measurements of Lzapp and DRC we substituted the values obtained in this study in the multiple regression equation of Rochester and coworkers. ${ }^{12}$ This equation $(\mathrm{LDI}=2 \times(0.984 \mathrm{Lzapp}+0.462 \mathrm{DRC}+34.8)$ in $\mathrm{mm} ; R^{2}=0.94$ ) was based on data from 16 subjects who had static radiographs taken during relaxation at various lung volumes over the full range of vital capacity. It gives the length of the right hemidiaphragm and we have doubled it for total diaphragm length. Figure 3 shows the relation between LDI measured directly from each radiograph and LDI derived from this equation by substituting Lzapp and DRC measured from the same radiographs. The derived values lie within the $95 \%$ confidence intervals for the equation with a relatively small scatter of points around the regression lines $\left(R^{2} 0.95\right.$ and 0.84 for subjects 1 and 2 respectively).

\section{MEASUREMENTS WITH ULTRASONOGRAPHY}

To determine whether the same pattern of change in Lzapp could be observed with a technique which did not require radiation exposure, the inspiratory and expulsive manoeuvres were repeated while Lzapp was continually monitored by ultrasonography (up to 28 images/second). A $12 \mathrm{~cm}$ linear array probe was oriented longitudinally in the mid axillary line. In both subjects an overt reduction was observed in Lzapp in the dynamic phase of strong inspiratory efforts performed near FRC. There was also a secondary increase in

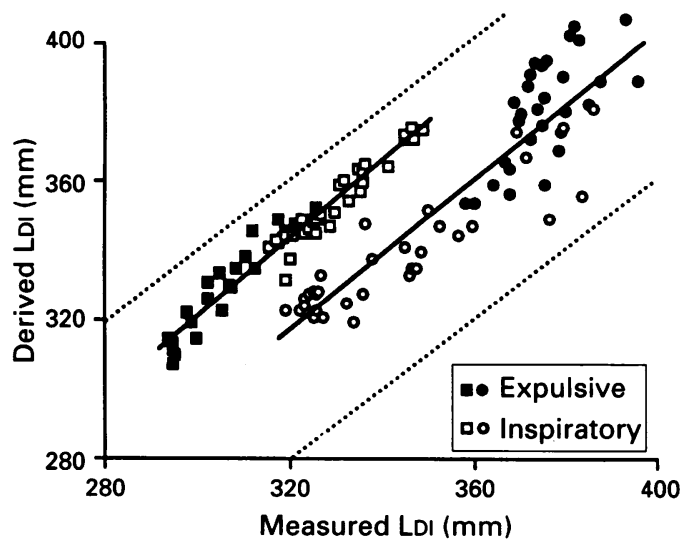

Figure 3 Relation between diaphragm length (LDI) measured directly from radiographs and derived by inserting values for Lzapp and DRC measured from the same radiographs into the multiple regression of

Rochester and colleagues (see table 4 in ref 12:

$L D I=2 \times(0.984 \mathrm{Lzapp}+0.462 \mathrm{DRC}+34.8) \mathrm{mm}$,

$\left.R^{2}=0.94, S E=0.98, n=70\right)$. All data points from three expulsive (closed symbols) and three inspiratory manoeuvres (open symbols) are shown for subject 1

(squares) and subject 2 (circles). They fall within the dotted lines which indicate the $95 \%$ confidence intervals of the multiple regression from ref 12 .
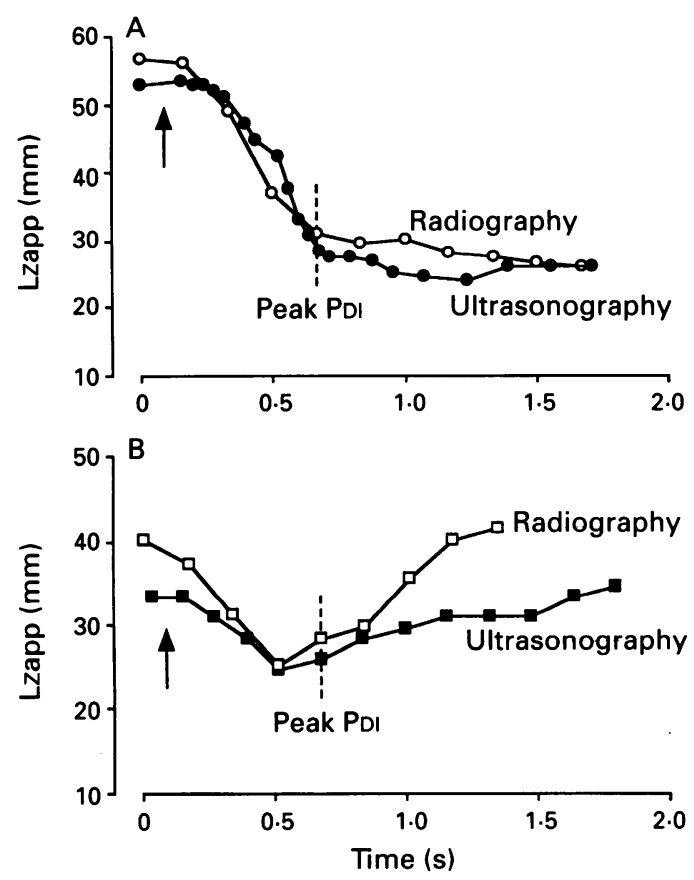

Figure 4 Data for one subject derived from radiographic (open symbols) and ultrasonographic measures (closed symbols) of Lzapp showing measurements of $(A)$ typical inspiratory and $(B)$ expulsive efforts. The arrows indicate the onset of the rise in PDI and the dashed lines indicate the time of peak PDI.

Lzapp during expulsive efforts at or before the peak PDI. Typical responses are compared for the two methods in fig 4. A formal comparison of the radiographic measurements of DRC and those obtained by magnetometers was not undertaken.

\section{Discussion}

Several studies have emphasised the likelihood of diaphragm length changes during the quasistatic manoeuvres used to test diaphragmatic strength. ${ }^{811} 16-19$ In a previous study we have shown that the diaphragm muscle fibres may shorten by $25 \%$ of their resting length during pressure development in a maximal inspiratory manoeuvre, while length changes at the onset of expulsive manoeuvres are biphasic. ${ }^{3}$ Because it has recently been shown that LDI can be predicted from measurements of Lzapp and DRC during relaxation at different lung volumes, ${ }^{12}$ the present study assessed this prediction during two of the voluntary manoeuvres commonly used to test respiratory muscle strength. A relation which holds during relaxation at different lung volumes, or during ventilation in an anaesthetised animal, may not apply to these maximal quasistatic manoeuvres which are associated with substantial distortions of rib cage and diaphragm shape.

Our results show that the relation between true LDI (measured) and LDI derived from measurements of Lzapp and DRC using the equation developed by Rochester and colleagues ${ }^{12}$ held in spite of differences between the two subjects in the maximal pressures achieved and in the rib cage movements associated with the manoeuvres.

The absolute values obtained for LDI, Lzapp, and DRC were slightly lower than those 
reported by Rochester and colleagues. ${ }^{12}$ The most likely explanation is a systematic difference in the method of measurement and scaling. The images $(10 \times 13.5 \mathrm{~cm})$ in the present study were measured by deriving Cartesian coordinates from a grid and application of a scaling factor, while Rochester and colleagues used a flexible tape measure on large films. No mention was made of correction for any magnification. Another potential source of error is the location of the insertion of diaphragmatic fibres: this was derived from anatomical landmarks in both studies. Such an error might influence the absolute values for LDI and Lzapp but not the changes during the dynamic manoeuvres. This problem would be less likely to occur with ultrasonographic measures (provided that a sufficiently long probe is used) because the origin of the costal fibres can be followed to the chest wall and viewed repeatedly during inspiratory efforts at TLC.

The predictive power of the multiple regression equation introduced by Rochester and colleagues was high under static conditions covering the full range of lung volumes. ${ }^{12}$ The novel result of our study was the ability of measurements of Lzapp and DRC to predict LDI accurately during dynamic respiratory manoeuvres in which other factors operate. These include rapid transient changes in diaphragmatic length and distortions of the diaphragmatic dome and rib cage. In spite of these limitations, however, the present data, combined with those of Rochester, ${ }^{12}$ show that more than $85 \%$ of the variance of the estimate of LDI is accounted for by Lzapp and DRC.

Digital sequential radiography was used here initially to provide quality images, although the radiation dose is unacceptable for routine application. Although the frame rate is higher with most videofluoroscopic units, the visual contrast is not sufficient to trace the diaphragm contour with confidence during dynamic manoeuvres (McKenzie and Gandevia, unpublished observations). However, it may be sufficient to obtain accurate measurements of Lzapp. ${ }^{13}$ In the latter study, using videofluoroscopy in dogs, it was necessary to use radio-opaque markers sewn onto the costal diaphragmatic surface to obtain LDI. To make the comparison of derived and actual LDI responses reported here it was necessary to have the best possible image quality. We could not justify exposing more healthy subjects than the two authors simply to determine the general applicability of the method. Rochester's work ${ }^{12}$ shows that the method is accurate for healthy subjects during relaxation. We have explored the physiological limits of the method in two healthy subjects. That $85 \%$ of the variance of LDI could be accounted for by Lzapp and DRC in two subjects who varied substantially in physical build and performance of the manoeuvres suggests that the technique may have broad application. It remains to be shown whether the technique will allow accurate derivation of LDI in patients, particularly in those with considerable hyperinflation.
It is already established that ultrasonography can be used to assess the thickness of the diaphragm. ${ }^{2021}$ Our results using ultrasonography show that it is possible to follow the dynamic changes in Lzapp with good time resolution and that these changes are quantitatively comparable to those observed radiographically. Thus, in healthy subjects, non-invasive measurements of Lzapp and DRC could be used to provide estimates of diaphragm length over a range of dynamic conditions. It is too early to predict a clinical application for this method of estimating LDI. It will certainly be possible in healthy subjects to normalise values of PDImax for changes in $\mathrm{LDI}^{3}$ and diaphragm thickness. ${ }^{21}$ Measurements of cross sectional area of the thorax may also help to convert measurements of pressure into estimates of muscle force or tension. ${ }^{2}$

This work was supportd by the National Health and Medical Research Council of Australia and the Asthma Foundation of New South Wales. We are grateful to the Department of Diagnostic Radiology and to Acuson Australia for the use of their equipment.

1 Laporta D, Grassino A. Assessment of transdiaphragmatic pressure in humans. $\mathcal{F}$ Appl Physiol 1985;58:1469-76.

2 Rochester DF, Arora NS, Braun NMT. Maximum contractile force of human diaphragm muscles determined in vivo. Trans Am Clin Climatol Assoc 1981;93:200-8.

3 Gandevia SC, Gorman RB, McKenzie DK, Southon FCG. Dynamic changes in human diaphragm length: maximal inspiratory and expulsive efforts studied with sequential radiography. $\mathcal{F}$ Physiol (Lond) 1992;457:167-76.

4 Edman KAP, Elzinga G, Noble MIM. Enhancement of mechanical performance by stretch during tetanic contractions of vertebrate skeletal muscle fibres. F Physiol (Lond) 1978;281:139-55.

5 Joyce GC, Rack MH, Westbury DR. The mechanical properties of cat soleus muscle during controlled lengthening and shortening movements. $\mathcal{f}$ Physiol (Lond) 1967;204:461-74.

$6 \mathrm{Katz} \mathrm{B}$. The relation between force and speed in muscular contraction. f Physiol (Lond) 1939;96:45-64.

7 Gandevia SC, McKenzie DK, Plassman BL. Activation of human respiratory muscles during different voluntary mahuman respiratory muscles during different vol

8 Hillman DR, Markos J, Finucane KE. Effect of abdominal compression on maximum transdiaphragmatic pressure. $\mathcal{f}$ Appl Physiol 1990;68:2296-304.

9 Braun NMT, Arora NS, Rochester DF. Force-length relationship of the normal human diaphragm. I Appl Physiol 1982;53:405-12.

10 Loring SH, Mead J, Griscom NT. Dependence of diaphragmatic length on lung volume and thoracoabdominal confguration. $\mathcal{F}$ Appl Physiol 1985;53:1961-70.

11 Mead J, Loring SH. Analysis of volume displacement and length changes of the diaphragm during breathing. $₹ \mathrm{Appl}$ Physiol 1982;53:750-5.

12 Petroll WM, Knight H, Rochester DF. Effect of lower rib cage expansion and diaphragm shortening on the zone of apposition. F Appl Physiol 1990;68:484-8.

13 Knight H, Petroll WM, Adams JM, Shaffer HA, Rochester DF. Videofluoroscopic assessment of muscle fiber shortening in the in situ canine diaphragm. I Appl Physio 1990;68:2200-7.

14 Agostoni E, Mognoni P. Deformation of the chest wall during breathing efforts. $f$ Appl Physiol 1966;21:1827-32.

15 Saunders NA, Kreitzer SM, Ingram RH. Rib cage deformation during static inspiratory efforts. $\mathcal{F}$ Appl Physiol 1979;46:1071-5.

16 Griffiths RI. Shortening of muscle fibres during stretch of the active cat medial gastrocnemius muscle: the role of
tendon compliance. $\mathcal{F}$ Physiol (Lond) 1991;436:219-36.

17 Griffiths RI, Shadwick RE. Properties of the tendons of the mammalian diaphragm. Proc Aust Physiol Pharmacol Soc 1990;21:55P.

18 Hubmayr RD, Sprung J, Nelson S. Determinants of transdiaphragmatic pressure in dogs. F Appl Physiol 1990 diaphragma

19 Sprung J, Deschamps C, Hubmayr RD, Walters BJ, Rodarte $\mathrm{JR}$. In vivo regional diaphragm function in dogs. $\mathcal{f} A p p l$ Physiol 1989;67:655-62.

20 Ueke J, De Bruin PF, Manzur A, Khan Y, Dubowitz V, Pride NB. In vivo assessment of diaphragm function by ultrasound in muscular dystrophy patients. Am Rev Respir Dis 1993;147:A693.

21 Cohn DB, Benditt JO, Hoppin Jr FG, Sherman CB, McCool FD. Diaphragm thickness: an index of inspiratory muscle strength. Am Rev Respir Dis 1993;147:A694. 